\title{
DIABETIC ACIDOSIS
}

\author{
AzMY R. Boutros, M.B., CH.B., D.A., F.F.A.R.C.S. ${ }^{*}$
}

AcrDosis is by far the most serious of all the complications of diabetes. ${ }^{1}$ Despite the marked lowering of its incidence and mortality among the diabetic population, it still deserves a great deal of attention from anyone dealing with diabetes in any way. Unfortunately, however, there is a tendency among anaesthetists and surgeons to rely completely on 'the internist to detect, assess, and tréat patients in severe diabetic acidosis. This inevitably leads to the deterioration of our knowledge and appreciation of the problems and disturbances involved in such cases. The anaesthetist may very well find himself confronted with such a problem, particularly in patients scheduled for emergency surgery, or in those who slip into diabetic coma postoperatively as a result of the anaesthesia, surgery, or the original pathology that necessitated the surgical intervention. Knowledge of the basic facts about diabetic acidosis can prove life-saving, or at least helpful in intelligently assessing the case with the internist.

\section{Definition of Diabetic Acriosis and Coma}

Although the word coma implies profound unconsciousness, a patient may be in grave danger from the rapidly developing ketosis long before he loses consciousness. A carbon dioxide combining power of $9 \mathrm{mEq} . / \mathrm{L}$. or less (normal $24 \mathrm{mEq} . / \mathrm{L}$.) is considered to be an indication of diabetic coma, whether the patient is actually unconscious or not. This, of course, is an arbitrary division, but it has the advantage of affording a criterion for further studies and classifications. Drowsiness and unconsciousness are actually due to the reaction of the brain to the combined effects of ketonaemia, acidaemia, dehydration, and electrolyte depletion.

\section{Precipitating Factors}

1. Errors in diet or insulin therapy.

2. Infections.

3. Stressful conditions, e.g., anaesthesia, surgery, or shock.

4. Severe vomiting or diarrhoea.

5. Thyrotoxicosis.

6. Pregnancy and toxaemias of pregnancy.

\section{Pathological Physiology of Diabettc Acidosis}

1. The initial metabolic defect is cessation of normal glucose metabolism. This will lead to the following:

"From the Department of Anaesthesia, University of Saskatchewan, and University Hospital, Saskatoon, Saskatchewan. Present address: Department of Anesthesia, State University of Iowa, and University Hospitals, Iowa City, Iowa, U.S.A. 
(a) Hyperglycaemia leading to a rise in the osmolarity of the extracellular compartment, which in turn leads to a shift of water from intracellular to extracellular compartments.

(b) Accumulation of ketone bodies (aceto-acetic acid, beta-hydroxybuteric acid, and acetone). This is a reflection of the failure of completeloxidation of two carbon fragments (active acetate) resulting from fatty acid oxidation.

The body defensive mechanisms will react to the added acids in the usual manner, ${ }^{2}$ thus:

(i) Plasma bicarbonate reacts with the adids giving rise to sodium salts of these acids, and to carbon dioxide, which stimulates the respiration. The end result is lowered total carbon dioxide content and marked lowering of the $\mathrm{CO}_{2}$ combining power (mainly bicarbonate) of the blood.

(ii) The renal phosphate mechanism will come into effect in an effort to counteract the general acidotic tendency of the blood.

(iii) Celullar cations (mainly potassium) will move to the extracellular compartment, thus increasing the cation content of the plasma. This, however, will lead to a marked loss of potassium from the body.

(iv) The ammonia mechanism of the kidneys contributes to the other body efforts in trying to eliminate excess $\mathrm{H}^{+}$while sparing other valuable cations. All these defensive mechanisms may still be inadequate, and a state of uncompensated acidosis ensues with a plasma bicarbonate of less than $9 \mathrm{mEq} . / \mathrm{L}$. and a $\mathrm{pH}$ as low as 6.8 (highest acidity compatible with life), severe dehydration and electrolyte depletion, renal failure and death.

(c) With the exhaustion of glycogen stores, protein is used for energy production purposes. This protein is supplied by the cells through protoplasmic catabolism, which will obviously cause the release of other cellular components as well, such as nitrogen, water, and electrolytes. These, in turn, will add to the plasma volume, increase the cardiac output, increase the glomerular filtration rate, and thus lead to polyuria and more water loss.

\section{Water and Electrolyte Disturbances}

(a) Water. It was estimated that a 70-kg. man in /severe diabetic acidosis would lose 6-7 litres of water. ${ }^{3,4}$ This is due to lack of intake and excessive loss of water through polyuria, vomiting, etc. Water loss is usually in excess of electrolyte loss because part of the solids lost is made up of glucose, organic acids, and catabolic end products.

(b) Sodium and chloride are lost essentially as normal saline.

(c) Potassium. At the onset of diabetic acidosis, the serum potassium level is normal, or even slightly elevated, probably owing to dehydration., ${ }^{4,6} \mathbf{6}$ Within a few hours, however, the potassium level falls precipitously, owing to the following reasons:

(i) Dilution by potassium-free fluids given intravenously.

(ii) Lack of intake.

(iii) Excessive loss in the urine.

(iv) Administration of insulin leading to the deposition of extracellular glucose, as glycogen, in the cells. This glycogen deposition entails the incorpora- 
tion of potassium in the molecule. It was estimated that deposition of 1 gram of glycogen requires about $0.36 \mathrm{mM}$. of potassium. ${ }^{7}$ This eventually leads to a noticeable shift of the cation from extracellular to intracellular compartments and marked reduction of serum potassium levels.

(d) Phosphates. This is the main intracellular anion, and it follows closely the changes that take place in potassium. On admission, there is usually some degree of hyperphosphataemia. This is soon replaced by hypophosphataemia once intensive fluid replacement is started. However, this hypophosphataemia does not seem to produce any significant clinical manifestations.

(e) Calcium. In diabetics with long-standing renal disease, low serum calcium may exist even before the onset of acidosis. With the further dilution resulting from liberal fluid replacement and the improvement of the $\mathrm{pH}$ towards alkalinity, the level of ionized calcium in the serum may fall to critical values,

\section{Clinical Manifestations of Diabetic Acidosis and Coma}

This subject is discussed in detail in most textbooks of medicine and only the salient points will be mentioned in this review.

1. History of insufficient insulin intake, infection, or any of the conditions that would precipitate acidosis.

2. Slow onset.

3. Thirst, polyuria, anorexia, abdominal pain, nausea, and vomiting.

4. Weakness, drowsiness, fever, and laboured respiration.

5. Dry florid skin, acetone smell in the breath, hypotension, and weak, rapid pulse.

6. Laboratory findings: (a) High blood sugar level, high blood ketone level, very low $\mathrm{CO}_{2}$ content in the plasma, and high leucocytic count. (b) Glycosuria, ketonuria, albuminuria, and granular casts in the urine.

\section{Treatment of Diabetic Acidosis and Cołia}

Although this is usually attended to by the physician, the, anaesthetist should familiarize himself with the measures used in these cases, and the rationale of the different lines of treatment.

The fact that a patient in diabetic acidosis is gravely ill and deteriorates very rapidly cannot be too strongly emphasized. The treatment must be energetic and in a hospital where all facilities for diagnosis and therapy are available.

Once the case is diagnosed as diabetic "acidosis, the following measures should be taken immediately:

\section{Insulin}

Regular or crystalline insulin, 50-100 units, should be given subcutaneously as soon as possible, the dose depending on the age of the patient and the severity of his condition. The same dose should be repeated every half-hour until there are signs of clinical and chemical improvement. It is extremely important to 
realize that as much insulin as is needed should be given in the first few hours of the treatment. The reasons for this urgency are as follows:

(a) Insulin resistance increases as acidosis advances.

(b) With the early reduction in blood sugar levels the harmful effects of increased osmolarity of the plasma and the resultant polyuria will be quickly checked.

The intravenous route may be used to give one dose of 50 units to achieve a greater speed of onset, otherwise the subcutaneous route is more effective and results in more sustained levels of insulin in the blood. However, if the patient is in circulatory collapse, absorption of insulin from subcutaneous sites may be eratic and unsatisfactory and the intravenous route is then preferred.

Once the condition of the patient shows adequate improvement, the subsequent doses of insulin can be based on an hourly or two-hourly urine analysis. Thus one could give 20 units for a red or orange Benedict reaction, 15 for yellow, and 10 for a yellowish green.

The total amount of insulin given in the first 24 hours may run into several thousand units, and one must always bear in mind the possibility that the patient might pass from a diabetic coma straight into a hypoglycaemic coma without ample warning. The only safeguards are to avoid giving any insulin if the Benedict test is green or blue, and to do frequent blood sugar estimations.

\section{Fluids}

As was mentioned before, a 70-kg. patient in diabetic acidosis may have a 6-7 litre water deficit. Correction of this dehydration is a matter of extreme urgency.

(a) Type of fluids. Physiological saline, or 0.85 per cent sodium chloride solution, is the most commonly used fluid. It is a very adequate solution, but not ideal because of the fact that it contains. equal amounts of sodium and chloride ( 145 meq./L. of each), while the fluid lost from the patient contains more sodium than chloride ( $\mathrm{Na} 145 \mathrm{meq} . / \mathrm{L}$. and $\mathrm{Cl} 110 \mathrm{meq} . / \mathrm{L}$ ). This excess chloride would unnecessarily increase the osmolarity of the plasma, increase the existing acidosis, and put a further excretory burden on the kidneys. A balanced mixture of sodium chloride and sodium lactate is a more suitable solution, as it contains less chloride than sodium.

Glucose solution should not be used in the early stages of treatment'because the added glucose will limit the benefits gained from the fluid replacement. Apart from that, extra glucose will only mean extra glycogen deposition when large doses of insulin are given simultaneously, and as was mentioned before, this will mean a shift of serum potassium to intracellular sites leading to marked reduction of serum potassium and even death from acute hypokalaemia. ${ }^{8}$ However, once the blood sugar levels approach normal figures, glucose can be given either by mouth or as 5 per cent solution in water intravenously.

(b) Quantity. 1500 to 2000 c.c. of normal saline should be given rapidly and should be repeated until the dehydration is corrected.

Rapid fluid replacement always carries the danger of overloading the cardiovascular system, resulting in acute pulmonary oedema or heart failure. However, 
this is not common in cases of diabetic acidosis and coma because of the extreme degree of dehydration present, and so long as the patient's condition is properly assessed and closely watched.

\section{Potassium}

The question of whether and when to give potassium to replace the loss is a controversial one. Definite hypokalaemia does exist, particularly 12-24 hours after intensive Hluid replacement and administration of massive doses of insulin have been started and there is no question that this deficit must be corrected. Some workers ${ }^{1}$ give potasșium chloride ( 1 gram per hour orally) only if and when changes suggestive of hypokalaemia appear in an electrocardiographic tracing. Others $8,9,10$ recommend the administration of potassium routinely to all patients in diabetic coma.

Serum potassium levels, as estimated by flame photometry, can be used to determine the time to give potassium. Thus a serum potassium level of less than $3 \mathrm{mEq}$./L. can be taken as an indication for giving potassium chloride or acetate or lactate. This can be given orally, as $50 \mathrm{mEq}$. $\mathrm{KCl}$ added to a glass of fruit juice, or else as $50-100 \mathrm{mEq}$. $\mathrm{KCl}$ intravenously in a litre of saline given over a twohour period, provided that the patient has adequate urinary flow.

\section{Gastric Lavage}

This is advantageous in most cases. It clears the fluid and old blood from the stomach, relieves abdominal discomfort, and stops, vomiting. It must be emphasized, however, that a patient in diabetic coma or acidosis is either comatose or at least drowsy, and gastric lavage in such a case will carry the same hazard of pulmonary aspiration as occurs whenever this technique is used in an unconscious or semiconscious patient, and it might be very wise, prior to the gastric lavage, to insert a cuffed endotracheal tube to seal off the trachea. If a patient's condition would not allow tracheal intubation, it is possible that gastric lavage would alsọ be contraindicated.

\section{Glucose and Food}

(a) In the first 3-5 hours glucose and food should be withheld for the reasons mentioned above.

(b) Following this period, when insulin has reduced the blood sugar level adequately, $10 \mathrm{gm}$. of glucose orally or intravenously could be given. This will replenish the carbohydrate stores and act as a safeguard against the production of hypoglycaemia. The food will help carbohydrate, protein, and fat metabolism to return to normal.

\section{Miscellaneous Measures}

(a) Vitamins: Various numbers of the Vitamin B complex act as essential enzymes for the processes involved in carbohydrate metabolism, and it seems reasonable to recommend giving these vitamins, particularly to patients in diabetic coma or acidosis who show some manifestations of neuropathy. 
(b) Antibiotics should be used whenever there is an infection, and sometimes even without obvious infection as a prophylactic measure.

(c) A cleansing enema helps to relieve distention.

\section{REFERENCES}

1. Joslin, E. P.; Root, H. F.; White, P.; \& Marble, A tus. 10th ed. Philadelphia: Lea \& Febiger (1959).

2. Wraterr, S. Applied Physiology. 9th ed. London: Oxford (1952).

3. Soskin, S. \& Levine, R. Physiological and Clinical Aspects of Ketosis. Am. J. Digest. Diseases 11: 305 (1944).

4. Nrchols, G. JR. \& Nrcrols, N. Changes in Tissue Composition during Acute Sodium -Depletion. Am. J. Physiol. 186: 383 (1956).

5. Nabarro, J. D. N.; Spencer, A. G. \& Stowers, J. M. Metabolic Studies in Severe Diabetic Ketosis. Quart. J. Med. 21: 225 (1952).

6. Seldin, D. W. \& TARAII, R. Metabolism of Glucose and Electrolytes in Diabetic Acidosis. J. Clin. Invest. 29: 552 (1950).

7. Darrow, D. C. Body Fluid Physiology: The Role of Potassium in Clinical Disturbances of Body Water and Electrolytes. N. Engl. J. Med. 242: 1014 (1950).

8. Perry, S. M. \& Rosenbaum, S. L. Hypopotassemia in Untreated Diabetic Coma. N. Engl. Med. 245: 847 (1951).

9. Martin, H. E. \& Wertman, M. Serum Potassium, Magniesium and Calcium Levels in Diabetic Acidosis. J. Clin. Invest. 26: 217 (1947).

10. Hoffman, W. S. Clinical Physiology of Potassium. J,A.M.A. 144: 1157, (1950). 